\title{
On Stability of Solutions to Equations Describing Incompressible Heat-Conducting Motions Under Navier's Boundary Conditions
}

\author{
Ewa Zadrzyńska ${ }^{1}$ • Wojciech M. Zajączkowski ${ }^{2,3}$
}

Received: 19 October 2015 / Accepted: 28 June 2017 / Published online: 3 August 2017

(C) The Author(s) 2017. This article is published with open access at Springerlink.com

\begin{abstract}
In this paper we prove existence of global strong-weak two-dimensional solutions to the Navier-Stokes and heat equations coupled by the external force dependent on temperature and the heat dissipation, respectively. The existence is proved in a bounded domain with the Navier boundary conditions for velocity and the Dirichlet boundary condition for temperature. Next, we prove existence of $3 \mathrm{~d}$ global strong solutions via stability.
\end{abstract}

Keywords Incompressible heat-conducting fluid - Stability of two-dimensional solution . Navier boundary conditions

\section{Introduction}

We consider a complete thermodynamical model of unsteady flows of incompressible homogeneous Newtonian fluids in a fixed bounded three-dimensional cylinder. The model comprises evolutionary equations for the velocity, pressure and temperature fields. We restrict our considerations to temperature independent material coefficients, that is, the viscosity and heat conductivity coefficients. We assume that the external force depends on temperature in a very special way (see Theorem 1). The heat equation contains the dissipative heating term which follows from the general theory of incompressible heat-conducting Navier-Stokes motions. We assume that the fluid is Newtonian, the internal energy is proportional to the temperature and the heat flux is proportional to the temperature gradient.

\footnotetext{
$凶$ W.M. Zaja̧czkowski

wz@impan.gov.pl

E. Zadrzyńska

emzad@mini.pw.edu.pl

1 Faculty of Mathematics and Information Sciences, Warsaw University of Technology, Koszykowa 75, 00-662 Warsaw, Poland

2 Institute of Mathematics, Polish Academy of Sciences, Śniadeckich 8, 00-656 Warsaw, Poland

3 Institute of Mathematics and Cryptology, Cybernetics Faculty, Military University of Technology, Kaliskiego 2, 00-908 Warsaw, Poland
} 
Let $D=\Omega \times(-a, a)$ be a bounded cylinder, where $\Omega \subset \mathbb{R}^{2}$ be an open domain, $L=2 a$ is the length of the cylinder which is parallel to the $x_{3}$-axis. The three-dimensional problem under the consideration is as follows

$$
\begin{array}{ll}
v_{t}+v \cdot \nabla v-v \operatorname{rot}^{2} v+\nabla p=\alpha(\theta) f & \text { in } D_{+}=D \times \mathbb{R}_{+}, \\
\operatorname{div} v=0 & \text { in } D_{+}, \\
\theta_{t}+v \cdot \nabla \theta-\varkappa \Delta \theta=v|\mathbb{D}(v)|^{2} & \text { in } D_{+}, \\
v \cdot \bar{n}=0 & \text { on } S_{+}=S \times \mathbb{R}_{+}, \\
\bar{n} \times \operatorname{rot} v=0 & \text { on } S_{+}, \\
\theta=\theta_{*} & \text { on } S_{+}, \\
\left.v\right|_{t=0}=v(0),\left.\quad \theta\right|_{t=0}=\theta(0) & \text { in } D,
\end{array}
$$

where $S=\partial D, v=\left(v_{1}(x, t), v_{2}(x, t), v_{3}(x, t)\right) \in \mathbb{R}^{3}$ is the velocity of the fluid, $x=$ $\left(x_{1}, x_{2}, x_{3}\right)$ are given Cartesian coordinates, $p=p(x, t) \in \mathbb{R}$ is the pressure, $\theta=\theta(x, t) \in$ $\mathbb{R}_{+}$is the temperature of the fluid, $f=\left(f_{1}(x, t), f_{2}(x, t), f_{3}(x, t)\right) \in \mathbb{R}^{3}$ is the external force field. The Cartesian coordinates $x$ are such that $x_{3}$-axis is parallel to the cylinder and it is located inside it. By the dot we denote the scalar product in $\mathbb{R}^{3}$.

By $\mathbb{D}(v)=\left\{v_{i, x_{j}}+v_{j, x_{j}}\right\}_{i, j=1,2,3}=\nabla v+\nabla v^{T}$ we denote the dilatation tensor, $\operatorname{rot}^{2}=$ rot rot $=-\Delta$ which holds on any divergence free vector. Moreover, $v>0$ is the constant viscosity coefficient, $\varkappa>0$ is also the constant heat conductivity coefficient and $\theta_{*}>0$ is a constant. Moreover, $\bar{n}$ is the unit outward normal vector to $S$. The boundary $S$ is split into two parts, i.e. $S=S_{1} \cup S_{2}$, where $S_{1}$ is parallel to the $x_{3}$-axis and $S_{2}$ is perpendicular to it. Additionally, $S_{2}=S_{2}(-a) \cup S_{2}(a)$, where $S_{2}(b)$ meets $x_{3}$-axis at $x_{3}=b$.

The function $\alpha=\alpha(\theta)$ from the r.h.s. of $(1.1)_{1}$ will be determined later. The term $\alpha(\theta) f$ generates the motion by heating and mechanically by the external force $f$. Similarly, the term $v|\mathbb{D}(v)|^{2}$ from the r.h.s. of $(1.1)_{3}$ heats the fluid by the viscous dissipation.

The aim of this paper is to prove the existence and uniqueness of a two-dimensional solution to problem (1), the global existence of sufficiently small three-dimensional perturbations of the 2d-solution and the existence of a global solution to problem (1) close to the two-dimensional solution assuming that the differences of external forces and initial data are sufficiently small in appropriate norms.

The important assumption is such that the external force $f$ does not decay in time. This implies that the solution does not vanish as time tends to infinity. Then, via stability and periodicity, the existence of stationary $2 \mathrm{~d}$ and $3 \mathrm{~d}$ solutions can be proved.

The stability of a two-dimensional solution to the Navier-Stokes system with a nondecaying in time external force has been examined in [21] and [23]. In [21] the system supplemented by the periodic boundary conditions is considered, while the paper [23] is devoted to the system in a cylinder with the Navier boundary conditions.

Problem (1) is much more difficult than the problem considered in [19], where the r.h.s. of $(1)_{3}$ is assumed to be zero. Then in [19] it is proved that there exists a global solution under the assumption that $v_{, x_{3}}(0), \theta_{, x_{3}}(0), f_{x_{3}}$ are sufficiently small in $L_{2}$-norms. Hence the obtained solution also has the same property. This means that the solution in [19] is close to the two-dimensional solution. However, in [19], contrary to this paper, it is not possible to control a behavior of solutions as time tends to infinity.

In paper [22] we proved the existence of a strong global solution to (1) $1,2,3,6,7$ with the slip boundary conditions for velocity which are close for all time to a two-dimensional solution. 
The above mentioned result is obtained under much stronger assumptions about $\alpha$ than these supposed in this paper. More precisely, we assumed in [22] that $|\alpha(\theta)|+\left|\alpha^{\prime}(\theta)\right| \leq \alpha_{*}$ for $\theta \geq \theta_{*}>0$, where $\alpha_{*}>0$ is a constant.

By a two-dimensional solution to problem (1) we mean functions $w=\left(w_{1}\left(x_{1}, x_{2}, t\right)\right.$, $\left.w_{2}\left(x_{1}, x_{2}, t\right), 0\right) \in \mathbb{R}^{2}, q=q\left(x_{1}, x_{2}, t\right) \in \mathbb{R}$ and $\vartheta=\vartheta\left(x_{1}, x_{2}, t\right) \in \mathbb{R}_{+}$which satisfy

$$
\begin{array}{ll}
w_{t}+w \cdot \nabla w+v \tilde{\operatorname{rot}}^{(2)} w+\nabla q=\alpha(\vartheta) h & \text { in } \Omega_{+} \equiv \Omega \times \mathbb{R}_{+}, \\
\operatorname{div} w=0 & \text { in } \Omega_{+}, \\
\vartheta_{t}+w \cdot \nabla \vartheta-\varkappa \Delta \vartheta=v|\mathbb{D}(w)|^{2} & \text { in } \Omega_{+}, \\
w \cdot \bar{n}=0 & \text { on } S_{0+}=S_{0} \times \mathbb{R}_{+}, \\
\operatorname{rot}^{(2)} w=0 & \text { on } S_{0+}, \\
\vartheta=\theta_{*} & \text { on } S_{0+}, \\
\left.w\right|_{t=0}=w(0),\left.\quad \vartheta\right|_{t=0}=\vartheta(0) & \text { in } \Omega,
\end{array}
$$

where $S_{0}$ is the boundary of $\Omega, S_{1}=S_{0} \times(-a, a), \operatorname{rot}^{(2)} w=w_{2, x_{1}}-w_{1, x_{2}}, \operatorname{rot} \varphi=$ $\left(\varphi_{, x_{2}},-\varphi_{, x_{1}}\right)$ and $h=\left(h_{1}\left(x_{1}, x_{2}, t\right), h_{2}\left(x_{1}, x_{2}, t\right), 0\right) \in \mathbb{R}^{2}$.

The direct calculation gives

$$
\operatorname{rot}^{2} w=\operatorname{rot}_{\operatorname{rot}^{(2)}} w
$$

Therefore the main operators in (1) $)_{1}$ and (2) $)_{1}$ are compatible. Moreover, it is shown in [23] that

$$
\bar{n} \times\left.\operatorname{rot} w\right|_{S_{0}}=\left.\bar{\tau} \operatorname{rot}^{(2)} w\right|_{S_{0}}
$$

and

$$
\bar{n} \times\left.\operatorname{rot} w\right|_{S_{2}}=0,
$$

where $\bar{n}=\left(n_{1}, n_{2}, 0\right), \bar{\tau}=\left(n_{2},-n_{1}, 0\right)$.

To show the stability of two-dimensional solutions of (2) we introduce the quantities

$$
u=v-w, \quad \eta=p-q, \quad \chi=\theta-\vartheta, \quad g=f-h
$$

which are solutions to the problem

$$
\begin{array}{ll}
u_{t}+u \cdot \nabla u+v \operatorname{rot}^{2} u+\nabla \eta=-w \cdot \nabla u-u \cdot \nabla w+[\alpha(\theta)-\alpha(\vartheta)] f+\alpha(\vartheta) g & \text { in } D_{+}, \\
\operatorname{div} u=0 & \text { in } D_{+}, \\
\chi_{t}+u \cdot \nabla \chi-\varkappa \Delta \chi=-w \cdot \nabla \chi-u \cdot \nabla \vartheta+v|\mathbb{D}(u)|^{2}+2 v \mathbb{D}(u): \mathbb{D}(w) & \text { in } D_{+}, \\
u \cdot \bar{n}=0 & \text { on } S_{+}, \\
\bar{n} \times \operatorname{rot} u=0 & \text { on } S_{+}, \\
\chi=0 & \text { on } S_{+}, \\
\left.u\right|_{t=0}=v(0)-w(0) \equiv u(0),\left.\quad \chi\right|_{t=0}=\theta(0)-\vartheta(0) \equiv \chi(0) & \text { in } \Omega .
\end{array}
$$


To formulate main results of the paper we introduce the following notation:

$$
\begin{aligned}
\bar{A}_{1}(T) & =\sup _{k \in \mathbb{N}_{0}} \int_{k T}^{(k+1) T}\|h(t)\|_{L_{2}(\Omega)}^{2} d t+\sup _{k \in \mathbb{N}_{0}} \int_{k T}^{(k+1) T}\|h(t)\|_{L_{\infty}(\Omega)}^{2 r} d t, \\
\bar{A}_{2} & =\|\operatorname{rot} w(0)\|_{L_{2}(\Omega)}^{2}+\|\vartheta(0)\|_{L_{2}(\Omega)}^{2},
\end{aligned}
$$

where $T>0, k \in \mathbb{N}_{0}=\mathbb{N} \cup\{0\}$.

The norms introduced above as well as norms occurring in formulations of the main results are defined in Sect. 2.

Theorem 1 Assume that $S_{0} \in C^{2}, 0<\sigma<\frac{1}{2}, T>0, h \in L_{2}\left(k T,(k+1) T ; L_{2}(\Omega)\right) \cap$ $L_{2 r}\left(k T,(k+1) T ; L_{\infty}(\Omega)\right)$ for all $k \in \mathbb{N}_{0}$, where $r=\frac{1}{1-2 \sigma}$ and $\bar{A}_{1}(T)<\infty$. Moreover, assume that $w(0) \in H^{1}(\Omega), \operatorname{div} w(0)=0,\left.w(0) \cdot \bar{n}\right|_{S_{0}}=0, \vartheta(0) \in L_{2}(\Omega), \vartheta(0) \geq \theta_{*}$ for some constant $\theta_{*}>0, \alpha \in C\left(\left[\theta_{*},+\infty\right)\right)$ and $|\alpha(\vartheta)| \leq a_{1}+a_{2} \vartheta^{\sigma}$ for $\vartheta \geq \theta_{*}$, where $a_{1} \geq 0$, $a_{2}>0$ are constants. Then there exists a unique solution

$$
\begin{aligned}
(w, \vartheta, q) \in & H^{2,1}(\Omega \times(k T,(k+1) T)) \times C\left([k T,(k+1) T] ; L_{2}(\Omega)\right) \\
& \cap L_{2}\left(k T,(k+1) T ; H^{1}(\Omega)\right) \times L_{2}\left(k T,(k+1) T ; H^{1}(\Omega)\right), \quad k \in \mathbb{N}_{0},
\end{aligned}
$$

to problem (2) with $\int_{\Omega} q d x=0$ for a.a. $t>0$. Moreover,

$$
\begin{aligned}
& \|w\|_{H^{2,1}(\Omega \times(k T,(k+1) T))}^{2}+\|\vartheta\|_{C\left([k T,(k+1) T] ; L_{2}(\Omega)\right)}^{2}+\|\vartheta\|_{L_{2}\left(k T,(k+1) T ; H^{1}(\Omega)\right)}^{2} \\
& \quad+\|q\|_{L_{2}\left(k T,(k+1) T ; H^{1}(\Omega)\right)}^{2} \leq c\left(\bar{A}_{1}, \bar{A}_{2}\right),
\end{aligned}
$$

where $c\left(\bar{A}_{1}, \bar{A}_{2}\right)$ does not depend on $k$.

Theorem 1 is proved in Sect. 3. The proof of Theorem 1 is based on the Faedo-Galerkin approximations combined with a step by step method. Since we need in the proof $H^{2}$ estimate for the velocity and $H^{1}$-estimate for the temperature, we assume that $|\alpha(\vartheta)| \leq a_{1}+$ $a_{2} \vartheta^{\sigma}$ for $\vartheta \geq \theta_{*}$, where $0<\sigma<\frac{1}{2}, a_{1}, a_{2}>0$. This restriction is generated by the dissipative heating term appearing in the r.h.s. of $(1)_{3}$. In the case of the lack of the dissipative term, the $L_{p}$-estimate for the temperature follows immediately, so the above restriction on $\alpha$ is not necessary. To obtain estimate (4) we show that the data in time intervals $[k T,(k+1) T]$, $k \in \mathbb{N}_{0}$ do not increase with $k$.

Our next result is the theorem about the stability of the two-dimensional solution obtained in Theorem 1 in the set of three-dimensional solutions.

Let $a>0$ be given. Then we define

$$
\begin{aligned}
\mathcal{M}= & \left\{(h, f) \in\left(L_{2, l o c}\left(\mathbb{R}_{+} ; L_{2}(\Omega)\right) \cap L_{2 r, l o c}\left(\mathbb{R}_{+} ; L_{\infty}(\Omega)\right)\right) \times L_{2, l o c}\left(\mathbb{R}_{+} ; L_{\infty}(D)\right):\right. \\
& \left.\exists T>0 \text { such that } T>a\left(\bar{A}_{1}(T)+\bar{A}_{2}+\sup _{k \in \mathbb{N}_{0}}\|f\|_{L_{2}\left(k T,(k+1) T ; L_{\infty}(D)\right.}\right)\right\},
\end{aligned}
$$

where $a=\frac{4 \tilde{c}_{2} \tilde{c}_{0}}{\tilde{c}_{1}}, \tilde{c}_{0}>0$ is the constant from (43) and $\tilde{c}_{1}, \tilde{c}_{2}>0$ are the constants from inequality (48). 
Theorem 2 Let $S_{0} \in C^{2}, 0<\sigma<\frac{1}{2}, h \in L_{2, l o c}\left(\mathbb{R}_{+} ; L_{2}(\Omega)\right) \cap L_{2 r, l o c}\left(\mathbb{R}_{+} ; L_{\infty}(\Omega)\right)$, where $r=\frac{1}{1-2 \sigma}$ and let $\bar{A}_{1}(T)<\infty$ for every $T>0$. Suppose that the assumptions of Theorem 1 concerning $w(0), \vartheta(0)$ and $\alpha$ are satisfied. Let $g \in C\left(\mathbb{R}_{+} ; L_{\infty}(D)\right), u(0) \in H^{1}(D)$, $\operatorname{div} u(0)=0,\left.u(0) \cdot \bar{n}\right|_{S}=0, \chi(0) \in L_{2}(D), \theta(0) \geq \theta_{*}, \alpha \in C^{1}\left(\left[\theta_{*},+\infty\right)\right),\left|\alpha^{\prime}(\theta)\right| \leq a_{3}$ for $\theta \geq \theta_{*}$, where $a_{3}>0$ is a constant. Assume that $(h, f) \in \mathcal{M}$. Moreover, suppose that

$$
\|\operatorname{rot} u(0)\|_{L_{2}(D)}^{2}+\|\chi(0)\|_{L_{2}(D E)}^{2} \leq \gamma
$$

and

$$
\|g(t)\|_{L_{\infty}(D)}^{2} \leq \delta \gamma \quad \text { for all } t \in \mathbb{R}_{+} .
$$

There exists $T>0$ such that if $\delta, \gamma>0$ are sufficiently small and if $(u, \chi, \eta) \in H^{2,1}(D \times$ $(k T,(k+1) T)) \times L_{2}\left(k T,(k+1) T ; H^{1}(D)\right) \times L_{2}\left(k T,(k+1) T ; H^{1}(D)\right)$ with $\chi_{t} \in$ $L_{2}\left(k T,(k+1) T ; H^{-1}(D)\right) k \in \mathbb{N}_{0}$, is a solution to problem (3) then

$$
\|\operatorname{rot} u(t)\|_{L_{2}(D)}^{2}+\|\chi(t)\|_{L_{2}(D)}^{2} \leq \bar{c} \gamma \quad \text { for all } t \in[k T,(k+1) T], \quad k \in \mathbb{N}_{0}
$$

and

$$
\begin{aligned}
& \|u\|_{H^{2,1}(D \times(k T,(k+1) T))}^{2}+\|\chi\|_{L_{2}\left(k T,(k+1) T ; H^{1}(D)\right)}^{2} \\
& \quad+\left\|\chi_{t}\right\|_{L_{2}\left(k T,(k+1) T ; H^{-1}(D)\right)}^{2}+\|\nabla \eta\|_{L_{2}\left(k T,(k+1) T ; L_{2}(D)\right)}^{2} \leq \bar{c} \gamma
\end{aligned}
$$

for all $k \in \mathbb{N}_{0}$,

where $\bar{c}=\bar{c}(T)$ does not depend on $k$ and $(w, \vartheta, q)$ is the two-dimensional solution to problem (1), which exists in virtue of Theorem 1.

To prove Theorem 2 we derive a nonlinear differential inequality (see the proof of Lemma 9) and then apply a contradiction argument.

Theorems 1 and 2 imply the following global existence result.

Theorem 3 Let $S_{0} \in C^{2}, 0<\sigma<\frac{1}{2}, h \in L_{2, l o c}\left(\mathbb{R}_{+} ; L_{2}(\Omega)\right) \cap L_{2 r, l o c}\left(\mathbb{R}_{+} ; L_{\infty}(\Omega)\right)$, where $r=\frac{1}{1-2 \sigma}$ and let $\bar{A}_{1}(T)<\infty$ for every $T>0$. Assume that the assumptions of Theorem 1 concerning $w(0), \vartheta(0)$ and $\alpha$ are satisfied. Let $v(0) \in H^{1}(D), \operatorname{div} v(0)=0$, $\left.v(0) \cdot \bar{n}\right|_{S}=0, \theta(0) \in L_{2}(D), \theta(0) \geq \theta_{*}, \alpha \in C^{1}\left(\left[\theta_{*},+\infty\right)\right),\left|\alpha^{\prime}(\theta)\right| \leq a_{3}$ for $\theta \geq \theta_{*}$, where $a_{3}>0$ is a constant. Moreover, assume that $f \in \mathcal{M}, g \in C\left(\mathbb{R}_{+} ; L_{\infty}(0)\right)$, and let conditions (5)-(6) be satisfied. There exists $T>0$ such that if $\delta$ and $\gamma$ are sufficiently small then there exists a unique global solution $(v, \theta, p) \in H^{2,1}(D \times(k T,(k+1) T)) \times C([k T,(k+$ 1) $\left.T] ; L_{2}(D)\right) \cap L_{2}\left(k T,(k+1) T ; H^{1}(D)\right) \times L_{2}\left(k T,(k+1) T ; H^{1}(D)\right), k \in \mathbb{N}_{0}$ of problem (1) with $\int_{\Omega} p d x=0$.

In view of Theorem 1 we prove Theorem 2 by using estimates (7)-(8) derived for the Faedo-Galerkin approximations.

There is a wide literature concerning equations describing motions of incompressible heat conducting fluids. Papers $[6,8,10,16,19]$ are concerned with solvability results for the Boussinesq equations, that is, for equations $(1)_{1,2,3}$, where $v|\mathbb{D}(v)|^{2}$ disappears. Kagei in [10] and Morimoto in [16] proved the existence of weak solutions to the three-dimensional Boussinesq equations with temperature - independent viscosity, while in [8] the weak solvability of the three-dimensional problem with temperature - dependent viscosity has been 
examined. Diaz and Galiano [6] showed existence of weak solutions to the following initialboundary value problem

$$
\begin{aligned}
& v_{t}+v \cdot \nabla v-\operatorname{div}(v(\theta) \mathbb{D}(v))=F(\theta), \quad \operatorname{div} v=0 \\
& \theta_{t}+v \cdot \nabla \theta-\Delta \varphi(\theta)=0 \\
& \left.v\right|_{S}=0,\left.\quad \varphi(\theta)\right|_{S}=\varphi_{D},
\end{aligned}
$$

under some restrictions on the functions $v(\theta), \varphi(\theta), F(\theta)$.

The question of the uniqueness of a solution to the two-dimensional Boussinesq equations with the Dirichlet boundary conditions and with temperature dependent viscosity has been studied in [9].

The recent papers concerning the Boussinesq system are [2] and [15]. In [2] Brandolese and Schonbek examine a large time behavior of both weak and strong solutions to the threedimensional system. Liu and $\mathrm{Li}$ [15] study the stability question for the Cauchy problem to equations of type (1), where the r.h.s. of $(1)_{3}$ vanishes and the r.h.s. of (1) equals $\theta \bar{e}_{3}, \bar{e}_{3}=$ $(0,0,1)$. They consider two solutions $\left(v_{1}, \theta_{1}\right)$ and $\left(v_{2}, \theta_{2}\right)$, where $\left\|\theta_{1}(0)\right\|_{L_{1}}$ and $\left\|\theta_{2}(0)\right\|_{L_{2}}+$ $\left\|v_{2}(0)\right\|_{B_{2,1}^{1 / 2}}$ are sufficiently small. The following stability is shown: $v_{1}-v_{2}, \theta_{1}-\theta_{2}$ are small if initially they are small. Since the Cauchy problem is considered, the technique of used function spaces is in the spirit of the French School. In contrast to our result solutions in [14] converge to zero as time tends to infinity.

Papers of Kagei $[11,12]$ are devoted to the two-dimensional system $(1)_{1,2,3}$ where the lefthand side of equation $(1)_{3}$ contains also the term $-e_{2} \cdot v, e_{2}=(0,1)$. Considering both the periodic and Dirichlet boundary conditions the author discusses the existence, uniqueness and large time behavior questions in dependence on assumptions about the right-hand side of $(1)_{1}$ and initial data. Kagei [11] touched the very difficult $2 d$ Bérnard convection problem. For a strongly nonhomogeneous boundary condition for the temperature the motion becomes very chaotic, so the concept of the strange attractor appears. The author relaxes the boundary condition on the temperature assuming that it is more homogeneous. Then he is able to derive many qualitative properties for the obtained weak solutions.

The Navier-Stokes system with temperature dependent viscosity coupled with the equation of conservation of internal energy has been studied in $[3,5,7,17]$.

Buliček, Feireisl, Málek [3] and Buliček, Kaplicky, Málek [4] proved the existence of suitable weak solutions to the Navier-Stokes-Fourier system in [3] and to the Stokes-Fourier in [4]. Moreover, Feireisl and Málek [7] studied the existence of weak solutions to the Navier-Stokes-Fourier system with temperature dependent viscosity. Naumann [17] proved the existence of weak solutions to system $(1)_{1}-(1)_{3}$ with the Dirichlet boundary conditions and with temperature-dependent viscosity and heat conductivity coefficients. Moreover, it is assumed that the r.h.s. of (1) disappears. Consighlieri, Rodrigues, Shilkin [5] consider problem similar to $(1)_{1}-(1)_{3}$ with temperature-dependent viscosity in 2 d-torus, that is, with periodic boundary conditions. The existence of weak solutions is proved. Moreover, the viscosity coefficient $v=v(\theta)$ satisfies $v_{1}, v_{2}, v_{1} \leq v(\theta) \leq v_{2}$, where $v_{1}, v_{2}$ are two positive constants. Kagei, Ružička, Thäter [13] proved the existence, uniqueness and stability of the motionless state for system of type (1), where $v \cdot \nabla v$ and $v \cdot \nabla \theta$ disappear. But in $(1)_{3}$ additionally appears the term $\theta v \cdot f$. They proved the existence of an unique solution in the class: $v \in C([0, T] ; D(P \Delta)), \theta \in C\left([0, T] ; H^{2}(\Omega) \cap H_{0}^{1}(\Omega)\right)$, where $P$ is the Helmholtz projection. Shilkin in [18] studies the classical solvability of the coupled system of two nonlinear parabolic equations with temperature-dependent viscosity which models 
a Poiseuille-type flow of an incompressible viscous fluid. He shows the existence of solutions to equations simpler than (1) because the terms $v \cdot \nabla v$ and $v \cdot \nabla \theta$ disappear. Moreover, it is supposed that $\alpha(\theta)=1$. The considered system is complemented by the Dirichlet conditions for the velocity and temperature. The problem is considered in a bounded domain.

The paper consists of four sections. In Sect. 2 notation and some auxiliary results are introduced. In Sect. 3 the two-dimensional problem is considered. Some useful a priori estimates are derived and Theorem 1 is proved. Section 4 contains the proofs of Theorems 2 and 3 .

\section{Notation and Auxiliary Results}

Let $\mathbb{N}_{0}=\mathbb{N} \cup\{0\}$ and let $\Omega \subset \mathbb{R}^{n}$ be an open set. By $L_{p}(\Omega), p \in[1, \infty]$ we denote the Lebesque space of integrable functions and by $W_{p}^{m}(\Omega), m \in \mathbb{N}_{0}, p \in[1, \infty]$ the Sobolev space. In the special case of $p=2$ we use the notation: $H^{m}(\Omega)=W_{2}^{m}(\Omega)$.

Let $I \subset \mathbb{R}$ be an open interval. Then $H^{2,1}(\Omega \times I)$ denotes the space of functions with the finite norm

$$
\|u\|_{H^{2,1}(\Omega \times I)}=\left(\left\|u_{t}\right\|_{L_{2}(\Omega \times I)}^{2}+\sum_{0 \leq|\alpha| \leq 2}\left\|D_{x}^{\alpha} u\right\|_{L_{2}(\Omega \times I)}^{2}\right)^{1 / 2},
$$

where $D_{x}^{\alpha}=\partial_{x_{1}}^{\alpha_{1}} \ldots \partial_{x_{n}}^{\alpha_{n}},|\alpha|=\alpha_{1}+\cdots+\alpha_{n}, \alpha_{i} \in \mathbb{N}_{0}, i=1, \ldots, n$.

Let $X$ be a Banach space. By $L_{p}(I ; X)$ we denote the space of all measurable functions $u: I \rightarrow X$ with the finite norm

$$
\|u\|_{L_{p}(I ; X)}=\left(\int_{I}\|u(t)\|_{X}^{p} d t\right)^{1 / p} \quad \text { if } 1<p<\infty
$$

and

$$
\|u\|_{L_{\infty}(I ; X)}=\operatorname{esssup}_{t \in I}\|u(t)\|_{X} .
$$

By $C(\bar{I} ; X)$ we denote the space of all continuous functions $u: \bar{I} \rightarrow X$ with the finite norm

$$
\|u\|_{C(\bar{I} ; X)}=\sup _{t \in I}\|u(t)\|_{X} \cdot
$$

Let $u=u\left(x_{1}, \ldots, x_{n}\right)$ be a scalar-valued function defined on $\Omega \subset \mathbb{R}^{n}, n=2,3$. Then by $\nabla u$ or $u_{x}$ we denote the gradient of $u$. For $u: \Omega \rightarrow \mathbb{R}^{n}, n=2,3$, by $\nabla u$ or $u_{x}$ we denote the matrix $\left[u_{i x_{j}}\right]_{i, j=1, \ldots, n}$. Moreover, then $\left|u_{x}\right|=\sqrt{\sum_{i j=1}^{n}\left|u_{i x_{j}}\right|^{2}}$.

We need the following lemma.

Lemma 1 Let $\theta(0) \in L_{2}(D)$ and assume that $\theta(0) \geq \theta_{*}$ for some $\theta_{*}>0$. Moreover, assume that functions $v, \theta$ such that $\nabla v \in L_{\frac{20}{7}}(D \times(k T,(k+1) T)), k \in \mathbb{N}_{0}, \theta \in L_{\frac{10}{3}}(D \times(k T,(k+$ 1)T)), $k \in \mathbb{N}_{0}$ satisfy (1). Then

$$
\theta \geq \theta_{*} \quad \text { a.e. in } D \times(k T,(k+1) T), k \in \mathbb{N}_{0} .
$$


Proof Multiply $(1)_{3}$ by $\left(\theta-\theta_{*}\right)_{-}=\min \left\{\theta-\theta_{*}, 0\right\}$. Then

$$
\begin{aligned}
& \frac{d}{d t} \int_{D}\left(\theta-\theta_{*}\right)_{-}^{2} d x+2 \varkappa \int_{D}\left|\nabla\left(\theta-\theta_{*}\right)_{-}\right|^{2} d x=2 v \int_{D}|\mathbb{D}(v)|^{2}\left(\theta-\theta_{*}\right)_{-} d x \leq 0 \\
& \quad \text { for all } t \in(k T,(k+1) T), \quad k \in \mathbb{N}_{0} .
\end{aligned}
$$

Integrating the above equality with respect to time yields

$$
\begin{aligned}
& \int_{D}\left(\theta-\theta_{*}\right)_{-}^{2} d x+2 \varkappa \int_{D \times(k T,(k+1) T)}\left|\nabla\left(\theta-\theta_{*}\right)_{-}\right|^{2} d x d t \\
& \quad=2 v \int_{D \times(k T,(k+1) T)}|\mathbb{D}(v)|^{2}\left(\theta-\theta_{*}\right)_{-} d x d t+\int_{D}\left(\theta(k T)-\theta_{*}\right)_{-}^{2} d x, \quad k \in \mathbb{N}_{0} .
\end{aligned}
$$

The 1.h.s. of the above equality implies that $\theta-\theta_{*} \in L_{10 / 3}(D \times(k T,(k+1) T))$. Then, by the Hölder inequality, the first integral on the r.h.s. is bounded if $\mathbb{D}(v) \in L_{20 / 7}(D \times(k T,(k+$ 1) $T)$ ).

Hence

$$
\int_{D}\left(\theta-\theta_{*}\right)_{-}^{2} d x \leq \int_{D}\left(\theta(k T)-\theta_{*}\right)_{-}^{2} d x=0,
$$

so $\left(\theta-\theta_{*}\right)_{-}=0$ a.e. in $D \times(k T,(k+1) T)$, which implies (9).

Consider the problem

$$
\begin{aligned}
w_{2 x_{1}}-w_{1 x_{2}}=b & \text { in } \Omega, \\
w_{1 x_{1}}+w_{2 x_{2}}=0 & \text { in } \Omega, \\
w \cdot \bar{n}=0 & \text { on } S_{0},
\end{aligned}
$$

where $\Omega \subset \mathbb{R}^{2}$ is an open domain, $S_{0}$ is the boundary of $\Omega$.

Lemma 2 (See [23]) Let $S_{0} \in C^{s+1}, b \in H^{s}(\Omega), s \in \mathbb{N}_{0}$. Then there exists a solution $w \in$ $H^{s+1}(\Omega)$ to problem $(10)$ and

$$
\|w\|_{H^{s+1}(\Omega)} \leq c\|b\|_{H^{s}(\Omega)} .
$$

Now, consider the elliptic overdetermined problem

$$
\begin{array}{ll}
\operatorname{rot} u=b & \text { in } D, \\
\operatorname{div} u=0 & \text { in } D, \\
u \cdot \bar{n}=0 & \text { on } S,
\end{array}
$$

where $D \subset \mathbb{R}^{3}$ is a cylinder.

Lemma 3 (See [20, 23]) Let $S_{1} \in C^{i+1}, b \in H^{i}(D), i=1,2, \operatorname{div} b=0$. Then there exists $a$ solution $u \in H^{i+1}(D)$ to problem (11) and

$$
\|u\|_{H^{i+1}(D)} \leq c\|b\|_{H^{i}(D)}, \quad i=0,1, \quad H^{0}(D)=L_{2}(D) .
$$


In Sect. 3 (see Lemma 5) we need the following lemma.

Lemma 4 Let $w$ be a sufficiently regular function satisfying

$$
\begin{gathered}
\operatorname{div} w=0 \quad \text { in } \Omega, \\
\operatorname{rot}^{(2)} w=0 \quad \text { on } S_{0} .
\end{gathered}
$$

Then

$$
\int_{\Omega} w \cdot \nabla w \cdot \operatorname{rot} \operatorname{rot} w^{(2)} d x=0 .
$$

Proof Since $\operatorname{rot}^{(2)} w=w_{2 x_{1}}-w_{1 x_{2}}$ and rot $\phi=\left(\phi_{x_{2}},-\phi_{x_{1}}\right)$, we have

$$
\begin{aligned}
\int_{\Omega} & w \cdot \nabla w \cdot \operatorname{rot} \operatorname{rot} w^{(2)} d x \\
= & \int_{\Omega}\left[w \cdot \nabla w_{1}\left(\operatorname{rot}^{(2)} w\right)_{x_{2}}-w \cdot \nabla w_{2}\left(\operatorname{rot}^{(2)} w\right)_{x_{1}}\right] d x \\
= & \int_{\Omega}\left[\left(w \cdot \nabla w_{1} \operatorname{rot}^{(2)} w\right)_{x_{2}}-\left(w \cdot \nabla w_{2} \operatorname{rot}^{(2)} w\right)_{x_{1}}\right] d x \\
& -\int_{\Omega}\left[\left(w \cdot \nabla w_{1}\right)_{x_{2}} \operatorname{rot}^{(2)} w-\left(w \cdot \nabla w_{2}\right)_{x_{1}} \operatorname{rot}^{(2)} w\right] d x \\
= & -\int_{S_{0}}\left(w \cdot \nabla w_{2} n_{1}-w \cdot \nabla w_{1} n_{2}\right) \operatorname{rot}^{(2)} w d S_{0} \\
& -\int_{\Omega}\left(w \cdot \nabla w_{1 x_{2}}-w \cdot \nabla w_{2 x_{1}}\right) \operatorname{rot}^{(2)} w d x \\
& -\int_{\Omega}\left(w_{1 x_{2}} w_{1 x_{1}}+w_{2 x_{2}} w_{1 x_{2}}-w_{1 x_{1}} w_{2 x_{1}}-w_{2 x_{1}} w_{2 x_{2}}\right) \operatorname{rot}^{(2)} w d x \\
\equiv & I_{1}+I_{2}+I_{3} .
\end{aligned}
$$

By condition $(12)_{2}$ we get that $I_{1}=0$. Next, by $(12)_{1}$

$$
\begin{aligned}
I_{2} & =\int_{\Omega} w \cdot \nabla \operatorname{rot}^{(2)} w \operatorname{rot}^{(2)} w d x=\frac{1}{2} \int_{\Omega} w \cdot \nabla\left(\operatorname{rot}^{(2)} w\right)^{2} d x \\
& =\frac{1}{2} \int_{S_{0}} w \cdot \bar{n}\left(\operatorname{rot}^{(2)} w\right)^{2} d S_{0}=0 .
\end{aligned}
$$

Finally,

$$
I_{3}=-\int_{\Omega} \operatorname{div} w\left(\operatorname{rot}^{(2)} w\right)^{2} d x=0
$$

This concludes the proof. 


\section{Existence of a Solution to Two-Dimensional Problem}

First, we derive a priori estimates for a two-dimensional solution to problem (2). Introduce the function $\bar{\vartheta}=\vartheta-\theta_{*}$. Then system (2) takes the form

$$
\begin{array}{ll}
w_{t}-w \cdot \nabla w+v \operatorname{rot}^{(2)} w+\nabla q=\alpha\left(\bar{\vartheta}+\theta_{*}\right) h & \text { in } \Omega_{+}, \\
\operatorname{div} w=0 & \text { in } \Omega_{+}, \\
\bar{\vartheta}_{t}+w \cdot \nabla \bar{\vartheta}-\varkappa \Delta \bar{\vartheta}=v|\mathbb{D}(w)|^{2} & \text { in } \Omega_{+}, \\
w \cdot \bar{n}=0 & \text { on } S_{0+}, \\
\operatorname{rot}^{(2)} w=0 & \text { on } S_{0+}, \\
\bar{\vartheta}=0 & \text { on } S_{0+}, \\
\left.w\right|_{t=0}=w(0),\left.\quad \bar{\vartheta}\right|_{t=0}=\bar{\vartheta}(0) & \text { in } \Omega,
\end{array}
$$

where $\bar{\vartheta}(0)=\vartheta(0)-\theta_{*}$. Assume that

$$
\vartheta(0) \geq \theta_{*} \text {. }
$$

Then $\vartheta \geq \theta_{*}$ a.e. in $\Omega_{+}$(see Lemma 1). Suppose also that

$$
\alpha \in C\left(\left[\theta_{*},+\infty\right)\right)
$$

and

$$
|\alpha(\vartheta)| \leq a_{1}+a_{2} \vartheta^{\sigma} \quad \text { for } \vartheta \geq \theta_{*},
$$

where $a_{1} \geq 0, a_{2}>0,0<\sigma<\frac{1}{2}$ are constants.

Introduce the notation

$$
\begin{aligned}
& X(t)=\|\operatorname{rot} w\|_{L_{2}(\Omega)}^{2}+\|\bar{\vartheta}\|_{L_{2}(\Omega)}, \\
& Y(t)=\|\operatorname{rot} w\|_{H^{1}(\Omega)}^{2}+\|\bar{\vartheta}\|_{L_{2}(\Omega)} .
\end{aligned}
$$

Lemma 5 Let $S_{0} \in C^{2}$ and $T>0$ be fixed. Assume that conditions (14)-(15) are satisfied and let $w(0) \in H^{1}(\Omega), \operatorname{div} w(0)=0,\left.w(0) \cdot \bar{n}\right|_{S_{0}}=0, \vartheta(0) \in L_{2}(\Omega)$. Moreover, let

$$
A_{1}=\sup _{k \in \mathbb{N}_{0}} \int_{k T}^{(k+1) T}\|h(t)\|_{L_{2}(\Omega)}^{2} d t+\sup _{k \in \mathbb{N}_{0}} \int_{k T}^{(k+1) T}\|h(t)\|_{L_{4}(\Omega)}^{2 r} d t<\infty,
$$

where $r=\frac{1}{1-2 \sigma}$. Assume that $(w, \bar{\vartheta}, q)$ is a sufficiently regular solution to problem (13). Then

$$
X(k T) \leq \frac{\bar{c}_{2} A_{1}}{1-e^{-\bar{c}_{1} T}}+X(0) \equiv A_{2},
$$

where $\bar{c}_{1}, \bar{c}_{2}>0$ are constants from inequality (21). Furthermore, 


$$
X(t)+\int_{k T}^{t} Y\left(t^{\prime}\right) d t^{\prime} \leq c\left(A_{1}+A_{2}\right)
$$

where $t \in(k T,(k+1) T], k \in \mathbb{N}_{0}, c>0$ is a constant.

Proof Multiplying equation (13) ${ }_{1}$ by $\operatorname{rot}^{\operatorname{rot}^{(2)}} w=-\Delta w$, integrating over $\Omega$ and using Lemma 2 yields

$$
\frac{1}{2} \frac{d}{d t}\|\operatorname{rot} w\|_{L_{2}(\Omega)}^{2}+v\|\operatorname{rot} w\|_{H^{1}(\Omega)}^{2}=-\int_{\Omega} \alpha\left(\bar{\vartheta}+\theta_{*}\right) h \Delta w d x .
$$

Using (15) we have

$$
\begin{aligned}
& \frac{d}{d t}\|\operatorname{rot} w\|_{L_{2}(\Omega)}^{2}+2 v\|\operatorname{rot} w\|_{H^{1}(\Omega)}^{2} \\
& \quad \leq c\left(\|h\|_{L_{4}(\Omega)}\|\bar{\vartheta}\|_{L_{4 \sigma}(\Omega)}^{\sigma}\|\Delta w\|_{L_{2}(\Omega)}+\|h\|_{L_{2}(\Omega)}\|\Delta w\|_{L_{2}(\Omega)}\right),
\end{aligned}
$$

where $\|\bar{\vartheta}\|_{L_{4 \vartheta}(\Omega)}=\left(\int_{\Omega} \bar{\vartheta}^{4 \sigma} d x\right)^{\frac{1}{4 \sigma}}$ for $0<\sigma<\frac{1}{2}$.

By the imbedding theorem, Young inequality and Lemma 3 we get

$$
\begin{aligned}
& \frac{d}{d t}\|\operatorname{rot} w\|_{L_{2}(\Omega)}^{2}+2 v\|\operatorname{rot} w\|_{H^{1}(\Omega)}^{2} \\
& \quad \leq \varepsilon\|\operatorname{rot} w\|_{H^{1}(\Omega)}^{2}+c(\varepsilon)\left(\|h\|_{L_{4}(\Omega)}^{2}\|\bar{\vartheta}\|_{L_{4 \sigma}(\Omega)}^{2 \sigma}+\|h\|_{L_{2}(\Omega)}^{2}\right) .
\end{aligned}
$$

Assuming that $\varepsilon$ is sufficiently small we obtain

$$
\frac{d}{d t}\|\operatorname{rot} w\|_{L_{2}(\Omega)}^{2}+c_{1}\|\operatorname{rot} w\|_{H^{1}(\Omega)}^{2} \leq c_{2}\left(\|h\|_{L_{4}(\Omega)}^{2}\|\bar{\vartheta}\|_{L_{2}(\Omega)}^{2 \sigma}+\|h\|_{L_{2}(\Omega)}^{2}\right) .
$$

Now, multiplying $(13)_{3}$ by $\bar{\vartheta}$ gives

$$
\frac{1}{2} \frac{d}{d t}\|\bar{\vartheta}\|_{L_{2}(\Omega)}^{2}+\varkappa \int_{\Omega}|\nabla \bar{\vartheta}|^{2} d x=v \int_{\Omega}|\mathbb{D}(w)|^{2} \bar{\vartheta} d x \leq c\|w\|_{H^{2}(\Omega)}^{2}\|\bar{\vartheta}\|_{L_{2}(\Omega)} .
$$

Using the Poincaré inequality and Lemma 2 we obtain

$$
\frac{d}{d t}\|\bar{\vartheta}\|_{L_{2}(\Omega)}+c_{3}\|\bar{\vartheta}\|_{L_{2}(\Omega)} \leq c\|w\|_{H^{2}(\Omega)}^{2} \leq c_{4}\|\operatorname{rot} w\|_{H^{1}(\Omega)}^{2}
$$

Multiplying (18) by a constant $c_{5}>0$ so large that $\frac{c_{1} c_{5}}{2}>c_{4}$ and adding (18) and (20) we get

$$
\begin{aligned}
& \frac{d}{d t}\left(\|\operatorname{rot} w\|_{L_{2}(\Omega)}^{2}+\|\bar{\vartheta}\|_{L_{2}(\Omega)}\right)+c\left(\|\operatorname{rot} w\|_{H^{1}(\Omega)}^{2}+\|\bar{\vartheta}\|_{L_{2}(\Omega)}\right) \\
& \quad \leq c\left(\|h\|_{L_{4}(\Omega)}^{2}\|\bar{\vartheta}\|_{L_{2}(\Omega)}^{2 \sigma}+\|h\|_{L_{2}(\Omega)}^{2}\right) \\
& \quad \leq \varepsilon\|\bar{\vartheta}\|_{L_{2}(\Omega)}+c(\varepsilon)\left(\|h\|_{L_{4}(\Omega)}^{2 r}+\|h\|_{L_{2}(\Omega)}^{2}\right)
\end{aligned}
$$


where we used that $2 \sigma<1$ and applied the Young inequality with $r^{\prime}=\frac{1}{2 \sigma}>1, r=\frac{r^{\prime}}{r^{\prime}-1}=$ $\frac{1}{1-2 \sigma}$. Choosing $\varepsilon$ sufficiently small we have

$$
\begin{aligned}
& \frac{d}{d t}\left(\|\operatorname{rot} w\|_{L_{2}(\Omega)}^{2}+\|\bar{\vartheta}\|_{L_{2}(\Omega)}\right)+\bar{c}_{1}\left(\|\operatorname{rot} w\|_{H^{1}(\Omega)}^{2}+\|\bar{\vartheta}\|_{L_{2}(\Omega)}\right) \\
& \quad \leq \bar{c}_{2}\left(\|h\|_{L_{4}(\Omega)}^{2 r}+\|h\|_{L_{2}(\Omega)}^{2}\right) .
\end{aligned}
$$

Hence

$$
\frac{d}{d t} X+\bar{c}_{1} X \leq \bar{c}_{2}\left(\|h\|_{L_{4}(\Omega)}^{2 r}+\|h\|_{L_{2}(\Omega)}^{2}\right)
$$

so

$$
\frac{d}{d t}\left(e^{\bar{c}_{1} t} X\right) \leq \bar{c}_{2} e^{\bar{c}_{1} t}\left(\|h\|_{L_{4}(\Omega)}^{2 r}+\|h\|_{L_{2}(\Omega)}^{2}\right) .
$$

Therefore,

$$
X(t) \leq \bar{c}_{2} \int_{k T}^{t}\left(\left\|h\left(t^{\prime}\right)\right\|_{L_{4}(\Omega)}^{2 r}+\left\|h\left(t^{\prime}\right)\right\|_{L_{2}(\Omega)}^{2}\right) d t^{\prime}+e^{-\bar{c}_{1}(t-k T)} X(k T)
$$

for $t \in(k T,(k+1) T], k \in \mathbb{N}_{0}$. Inserting $t=(k+1) T$ to (22) implies

$$
X((k+1) T) \leq \bar{c}_{1} \int_{k T}^{(k+1) T}\left(\left\|h\left(t^{\prime}\right)\right\|_{L_{4}(\Omega)}^{2 r}+\left\|h\left(t^{\prime}\right)\right\|_{L_{2}(\Omega)}^{2}\right) d t^{\prime}+e^{-\bar{c}_{1} T} X(k T) .
$$

Therefore, by iteration inequality (16) follows. Moreover, integrating (21) over $(k T, t)$ and using (16), we get (17).

Lemma 6 Let the assumptions of Lemma 5 hold. Then

$$
\sup _{k T \leq t \leq(k+1) T}\|w(t)\|_{H^{1}(\Omega)}^{2} \leq c\left(A_{1}+A_{2}\right) \quad \text { for } k \in \mathbb{N}_{0}
$$

and

$$
\|w\|_{L_{2}\left(k T, t ; H^{2}(\Omega)\right)}^{2}+\|\bar{\vartheta}\|_{L_{2}\left(k T, t ; H^{1}(\Omega)\right)}^{2} \leq c\left(A_{1}+A_{2}\right) \quad \text { for } t \in(k T,(k+1) T], k \in \mathbb{N}_{0},
$$

where $c>0$ does not depend on $k$.

Proof Estimate $\|w\|_{H^{1}(\Omega)}^{2} \leq c\|\operatorname{rot} w\|_{L_{2}(\Omega)}^{2}$ (see Lemma 2) and (17) imply (23). Next, by the estimate

$$
\|w\|_{H^{2}(\Omega)}^{2} \leq c\|\operatorname{rot} w\|_{H^{1}(\Omega)}^{2}
$$

we get

$$
\|w\|_{L_{2}\left(k T, t ; H^{2}(\Omega)\right)}^{2} \leq c\left(A_{1}+A_{2}\right) .
$$

Now, integrating (19) over $(k T, t), t \in(k T,(k+1) T], k \in \mathbb{N}_{0}$ and using (17) and (25) we obtain 


$$
\|\bar{\vartheta}\|_{L_{2}\left(k T, t ; H^{1}(\Omega)\right)}^{2} \leq c\left(A_{1}+A_{2}\right) .
$$

This ends the proof.

Lemma 7 Let the assumptions of Lemma 5 be satisfied. Let $(w, \bar{\vartheta}, q)$ be a sufficiently regular solution to problem (13). Then

$$
\left\|w_{t}\right\|_{L_{2}(\Omega \times(k T,(k+1) T))}^{2}+\left\|\vartheta_{t}\right\|_{L_{2}\left(k T,(k+1) T ; H^{-1}(\Omega)\right)}^{2}+\|\nabla q\|_{L_{2}(\Omega \times(k T,(k+1) T))}^{2} \leq c\left(A_{1}^{2}, A_{2}^{2}\right),
$$

where $c$ does not depend on $k$.

Proof Multiply (13) $)_{1}$ by $w_{t}$ and integrate over $\Omega$ and with respect to time from $k T$ to $(k+1) T$. We get

$$
\begin{aligned}
& \left\|w_{t}\right\|_{L_{2}(\Omega \times(k T,(k+1) T))}^{2}+\int_{\Omega}\left|\operatorname{rot}^{(2)} w((k+1) T)\right|^{2} d x \\
& \leq c\left(\int_{k T}^{(k+1) T} \int_{\Omega}\left|\alpha\left(\bar{\vartheta}+\theta_{*}\right) h w_{t}\right| d x d t+\int_{k T}^{(k+1) T} \int_{\Omega}\left|w \cdot \nabla w \cdot w_{t}\right| d x d t\right. \\
& \left.\quad+\int_{\Omega}\left|\operatorname{rot}^{(2)} w(k T)\right|^{2} d x\right) \leq \varepsilon\left\|w_{t}\right\|_{L_{2}(\Omega \times(k T,(k+1) T))}^{2} \\
& \quad+c\left(\int_{k T}^{(k+1) T}\|h\|_{L_{4}(\Omega)}\|\bar{\vartheta}\|_{L_{2}(\Omega)}^{\sigma}\left\|w_{t}\right\|_{L_{2}(\Omega)} d t+\int_{k T}^{(k+1) T}\|h\|_{L_{2}(\Omega)}\left\|w_{t}\right\|_{L_{2}(\Omega)} d t\right) \\
& \quad+c(\varepsilon)\left(\int_{k T}^{(k+1) T} \int_{\Omega}|w|^{2}\left|w_{x}\right|^{2} d x d t+\int_{\Omega}\left|\operatorname{rot}^{(2)} w(k T)\right|^{2} d x\right) .
\end{aligned}
$$

Using in (26) the estimate

$$
\begin{aligned}
& \int_{k T}^{(k+1) T}\|h\|_{L_{4}(\Omega)}\|\bar{\vartheta}\|_{L_{2}(\Omega)}^{\sigma}\left\|w_{t}\right\|_{L_{2}(\Omega)} d t \\
& \quad \leq \varepsilon\left\|w_{t}\right\|_{L_{2}(\Omega \times(k T,(k+1) T))}^{2}+c(\varepsilon) \sup _{k T \leq t \leq(k+1) T}\|\bar{\vartheta}\|_{L_{2}(\Omega)}^{2 \sigma} \int_{k T}^{(k+1) T}\|h\|_{L_{4}(\Omega)}^{2} d t,
\end{aligned}
$$

next applying Lemmas 5-6 and assuming that $\varepsilon$ is sufficiently small we obtain

$$
\left\|w_{t}\right\|_{L_{2}(\Omega \times(k T,(k+1) T))}^{2} \leq c\left(A_{1}, A_{2}\right) .
$$

Next, (13) 1 yields

$$
\begin{aligned}
& \|\nabla q\|_{L_{2}(\Omega \times(k T,(k+1) T))}^{2} \\
& \quad \leq c\left(\left\|w_{t}\right\|_{L_{2}(\Omega \times(k T,(k+1) T))}^{2}+\|w\|_{L_{2}\left(k T,(k+1) T ; H^{2}(\Omega)\right)}^{2}\right.
\end{aligned}
$$




$$
\begin{aligned}
& \quad+\|w\|_{C\left([k T,(k+1) T] ; H^{1}(\Omega)\right)}^{2} \cdot\|w\|_{L_{2}\left(k T,(k+1) T ; H^{2}(\Omega)\right)}^{2}+\|h\|_{L_{2}\left(k T,(k+1) T ; L_{2}(\Omega)\right)}^{2} \\
& \left.\quad+\|\bar{\vartheta}\|_{L_{\infty}\left([k T,(k+1) T] ; L_{2}(\Omega)\right)}^{2 \sigma}\|h\|_{L_{2}\left(k T,(k+1) T ; L_{4}(\Omega)\right)}^{2}\right) \\
& \leq c\left(A_{1}, A_{2}\right) .
\end{aligned}
$$

Finally, equation (13) 3 and Lemmas 5-6 imply

$$
\begin{aligned}
& \left\|\bar{\vartheta}_{t}\right\|_{L_{2}\left(k T,(k+1) T ; H^{-1}(\Omega)\right)}^{2} \\
& \leq c\left(\|\bar{\vartheta}\|_{L_{2}\left(k T,(k+1) T ; H^{1}(\Omega)\right)}^{2}+\int_{k T}^{(k+1) T}\|w\|_{L_{4}(\Omega)}^{2}\left\|\bar{\vartheta}_{x}\right\|_{L_{4}(\Omega)}^{2} d t\right. \\
& \left.\quad+\int_{k T}^{(k+1) T}\left\|w_{x}\right\|_{L_{4}(\Omega)}^{2}\left\|w_{x}\right\|_{L_{2}(\Omega)}^{2} d t\right) \\
& \leq c\left[\|\bar{\vartheta}\|_{L_{2}\left(k T,(k+1) T ; H^{1}(\Omega)\right)}^{2}+\|w\|_{C\left([k T,(k+1) T] ; H^{1}(\Omega)\right)}^{2}\left(\|\bar{\vartheta}\|_{L_{2}\left(k T,(k+1) T ; H^{1}(\Omega)\right)}^{2}\right.\right. \\
& \left.\left.\quad+\|w\|_{L_{2}\left(k T,(k+1) T ; H^{2}(\Omega)\right)}^{2}\right)\right] \\
& \leq c\left(A_{1}, A_{2}\right) .
\end{aligned}
$$

This ends the proof.

Now, our goal is to prove the existence of a strong-weak solution to problem (13). Denote

$$
V=\text { the closure of }\left\{v \in C^{\infty}(\bar{\Omega}): \operatorname{div} v=0, v \cdot \bar{n}=0 \text { on } S_{0}\right\} \quad \text { in } H^{1}(\Omega) .
$$

Definition 1 Let $T>0$ be given. We call a function $(w, \bar{\vartheta})$ a weak solution to problem (13) if $w \in L_{\infty}\left(k T,(k+1) T ; L_{2}(\Omega)\right) \cap L_{2}(k T,(k+1) T ; V), \bar{\vartheta} \in L_{\infty}\left(k T,(k+1) T ; L_{2}(\Omega)\right) \cap$ $L_{2}\left(k T,(k+1) T ; H_{0}^{1}(\Omega)\right), w_{t} \in L_{2}\left(k T,(k+1) T ; V^{*}\right), \bar{\vartheta}_{t} \in L_{2}\left(k T,(k+1) T ; H^{-1}(\Omega)\right)$ for all $k \in \mathbb{N}_{0}$ and

$$
\begin{aligned}
& \frac{d}{d t} \int_{\Omega} w \cdot \phi d x+v \int_{\Omega} \operatorname{rot}^{(2)} w \cdot \operatorname{rot}^{(2)} \phi d x+\int_{\Omega} w \cdot \nabla w \cdot \phi d x \\
& \quad=\int_{\Omega} \alpha\left(\bar{\vartheta}+\theta_{*}\right) h \cdot \phi d x \quad \text { for every } \phi \in V,
\end{aligned}
$$

in the sense of distributions on $(k T,(k+1) T)$,

$$
\begin{aligned}
& \frac{d}{d t} \int_{\Omega} \bar{\vartheta} \psi d x+\varkappa \int_{\Omega} \nabla \bar{\vartheta} \cdot \nabla \psi d x+\int_{\Omega} w \cdot \nabla \bar{\vartheta} \psi d x \\
& \quad=v \int_{\Omega}|\mathbb{D}(w)|^{2} \psi d x \quad \text { for every } \psi \in H_{0}^{1}(\Omega),
\end{aligned}
$$

in the sense of distributions on $(k T,(k+1) T)$,

$$
\left.w\right|_{t=k T}=w(k T),
$$




$$
\left.\bar{\vartheta}\right|_{t=k T}=\bar{\vartheta}(k T)
$$

for all $k \in \mathbb{N}_{0}$.

Definition 2 The triple $(w, q, \bar{\vartheta})$ is called a strong-weak solution to problem (13) if $(w, \bar{\vartheta})$ is a weak solution to (13) and if additionally $w \in L_{\infty}\left(k T,(k+1) T ; H^{1}(\Omega)\right) \cap L_{2}(k T,(k+$ 1) $\left.T ; H^{2}(\Omega)\right), \nabla q \in L_{2}\left(k T,(k+1) T ; L_{2}(\Omega)\right)$ for all $k \in \mathbb{N}_{0}$.

Now, we can sketch the proof of Theorem 1.

Proof of Theorem 1 We prove the existence of a strong-weak solution to problem (13) by applying the Faedo-Galerkin approximations. Let $\left\{\phi_{i}\right\}_{i=1}^{\infty}$ and $\left\{\psi_{i}\right\}_{i=1}^{\infty}$ be bases in $V$ and $H_{0}^{1}(\Omega)$, respectively. For each $m \in \mathbb{N}$, we define an approximate solution $w_{m}(t)=$ $\sum_{i=1}^{m} c_{i m}(t) \phi_{i}, \bar{\vartheta}_{m}(t)=\sum_{i=1}^{m} d_{i m}(t) \psi_{i}$, which satisfies the following system of ordinary differential equations

$$
\begin{aligned}
& \int_{\Omega} w_{m}^{\prime}(t) \cdot \phi_{j} d x+v \int_{\Omega} \operatorname{rot}^{(2)} w_{m}(t) \cdot \operatorname{rot}^{(2)} \phi_{j} d x+\int_{\Omega} w_{m}(t) \cdot \nabla w_{m}(t) \cdot \phi_{j} d x \\
& \quad=\int_{\Omega} \alpha\left(\bar{\vartheta}_{m}(t)+\theta_{*}\right) h(t) \cdot \phi_{j} d x \\
& \quad \text { for } j=1, \ldots, m, \quad t \in[k T,(k+1) T], \quad k \in \mathbb{N}_{0}, \\
& \int_{\Omega} \bar{\vartheta}_{m}^{\prime}(t) \psi_{j} d x+\varkappa \int_{\Omega} \nabla \bar{\vartheta}_{m}(t) \cdot \nabla \psi_{j} d x+\int_{\Omega} w_{m}(t) \cdot \nabla \bar{\vartheta}_{m}(t) \psi_{j} d x \\
& \quad=v \int_{\Omega}\left|\mathbb{D}\left(w_{m}(t)\right)\right|^{2} \psi_{j} d x \quad \text { for } j=1, \ldots, m, t \in[k T,(k+1) T], k \in \mathbb{N}_{0}, \\
& \left.w_{m}\right|_{t=k T}=w_{k T m}, \\
& \left.\bar{\vartheta}_{m}\right|_{t=k T}=\bar{\vartheta}_{k T m},
\end{aligned}
$$

where $w_{k T m} \in \operatorname{span}\left\{\phi_{1}, \ldots, \phi_{m}\right\}, w_{k T m} \rightarrow w(k T)$ strongly in $V$ as $m \rightarrow \infty, \bar{\vartheta}_{k T m} \in$ $\operatorname{span}\left\{\psi_{1}, \ldots, \psi_{m}\right\}, \bar{\vartheta}_{k T m} \rightarrow \bar{\vartheta}(k T)$ strongly in $L^{2}(\Omega)$.

We choose a special basis $\left\{\phi_{j}\right\}_{j=1}^{\infty}$ which consists of the eigenfunctions of the Stokes system with Navier's boundary conditions, that is, $\phi_{j}$ and some $p_{j}$ satisfy

$$
\begin{array}{ll}
-v \Delta \phi_{j}+\nabla p_{j}=\lambda_{j} \phi_{j} & \text { in } \Omega, \\
\operatorname{div} \phi_{j}=0 & \text { in } \Omega, \\
\phi_{j} \cdot \bar{n}=0 & \text { on } S_{0}, \\
\operatorname{rot} \phi_{j} \times \bar{n}=0 & \text { on } S_{0},
\end{array}
$$

where $\lambda_{j} \rightarrow \infty$ as $j \rightarrow \infty$. In what follows we will use the weak formulation of (29), that is,

$$
v \int_{\Omega} \operatorname{rot} \phi_{j} \cdot \operatorname{rot} v d x=\lambda_{j} \int_{\Omega} \phi_{j} \cdot v d x \quad \forall v \in V, \quad j=1,2, \ldots
$$


Now, we multiply the both sides of (27) by $\lambda_{j}$ and use (30) to the first and second term on the left-hand side of (27). We also use the equality $\int_{\Omega} \operatorname{rot} w_{m}(t) \cdot \operatorname{rot}^{(2)} \phi_{j} d x=\int_{\Omega} \operatorname{rot} w_{m}(t)$. $\operatorname{rot} \phi_{j} d x$. To the other two terms in (27) we apply (30) and the identity

$$
\int_{\Omega} \operatorname{rot} \phi_{j} \cdot \operatorname{rot} v d x=-\int_{\Omega} v \cdot \operatorname{rot}^{2} \phi_{j} d x \quad \forall v \in V .
$$

Next, multiplying the resulting system of equations by $c_{j m}$ and summing for $j=1, \ldots, m$ we get

$$
\frac{1}{2} \frac{d}{d t}\left\|\operatorname{rot} w_{m}\right\|_{L_{2}(\Omega)}^{2}+v\left\|\operatorname{rot} w_{m}\right\|_{H^{1}(\Omega)}^{2}=-\int_{\Omega} \alpha\left(\bar{\vartheta}_{m}+\theta_{*}\right) h \operatorname{rot}^{2} w_{m} d x
$$

where we used that by Lemma 4

$$
\int_{\Omega} w_{m}(t) \cdot \nabla w_{m}(t) \cdot \operatorname{rot}^{2} w_{m}(t) d x=0 .
$$

Similarly, multiplying (28) by $d_{j m}(t)$ and summing for $j=1, \ldots, m$ we obtain

$$
\frac{1}{2} \frac{d}{d t}\left\|\bar{\vartheta}_{m}\right\|_{L_{2}(\Omega)}^{2}+\varkappa \int_{\Omega}\left|\nabla \bar{\vartheta}_{m}\right|^{2} d x=v \int_{\Omega}\left|\mathbb{D}\left(w_{m}\right)\right|^{2} \bar{\vartheta}_{m} d x .
$$

Then repeating the considerations from the proofs of Lemmas 5-7 we derive the following estimate

$$
\begin{aligned}
\| w_{m} & \left\|_{L_{\infty}(k T,(k+1) T ; V)}+\right\| w_{m} \|_{L_{2}\left(k T,(k+1) T ; H^{2}(\Omega)\right)} \\
& +\left\|\bar{\vartheta}_{m}\right\|_{L_{\infty}\left(k T,(k+1) T ; L_{2}(\Omega)\right)}+\left\|\bar{\vartheta}_{m}\right\|_{L_{2}\left(k T,(k+1) T ; H_{0}^{1}(\Omega)\right)} \\
& +\left\|w_{m t}\right\|_{L_{2}\left(k T,(k+1) T ; L_{2}(\Omega)\right)}+\left\|\bar{\vartheta}_{m t}\right\|_{L_{2}\left(k T,(k+1) T ; H^{-1}(\Omega)\right)} \\
\leq & c\left(A_{1}, A_{2}\right)
\end{aligned}
$$

where $c\left(A_{1}, A_{2}\right)$ does not depend on $k$. By (31) and the Aubin-Lions lemma (see [14], Theorem 5.1) there exist subsequences of $w_{m}$ and $\bar{\vartheta}_{m}$ still denoted by $w_{m}$ and $\bar{\vartheta}_{m}$ such that

$$
\begin{aligned}
w_{m} \rightarrow & w \text { weakly in } L_{2}\left(k T,(k+1) T ; H^{2}(\Omega)\right) \\
& \text { and } * \text {-weakly in } L_{\infty}(k T,(k+1) T ; V), \\
w_{m t} \rightarrow & w_{t} \text { weakly in } L_{2}\left(k T,(k+1) T ; L_{2}(\Omega)\right), \\
w_{m} \rightarrow & w \text { strongly in } L_{2}\left(k T,(k+1) T ; H^{1}(\Omega)\right), \\
\bar{\vartheta}_{m} \rightarrow & \bar{\vartheta} \text { weakly in } L_{2}\left(k T,(k+1) T ; H_{0}^{1}(\Omega)\right) \\
& \text { and *-weakly in } L_{\infty}\left(k T,(k+1) T ; L_{2}(\Omega)\right), \\
\bar{\vartheta}_{m t} \rightarrow & \bar{\vartheta}_{t} \text { weakly in } L_{2}\left(k T,(k+1) T ; H^{-1}(\Omega)\right), \\
\bar{\vartheta}_{m} \rightarrow & \bar{\vartheta} \text { strongly in } L_{2}\left(k T,(k+1) T ; L_{2}(\Omega)\right) .
\end{aligned}
$$


The above convergences enable passing to the limit in (27)-(28) in the standard way (after multiplying (27)-(28) by appropriate functions of $t$ and integrating them in $[k T,(k+1) T])$. In particular passing to the limit in the term on the r.h.s. of (27) is possible thanks to the strong convergence of $\bar{\vartheta}_{m}$ in $L_{2}\left(k T,(k+1) T ; L_{2}(\Omega)\right)$, which yields that for a subsequence of $\bar{\vartheta}_{m}$ we have

$$
\bar{\vartheta}_{m} \rightarrow \bar{\vartheta} \quad \text { a.e. in } \Omega \times(k T,(k+1) T) .
$$

Therefore,

$$
\alpha\left(\bar{\vartheta}_{m}+\theta_{*}\right) \rightarrow \alpha\left(\bar{\vartheta}+\theta_{*}\right) \quad \text { a.e. in } \Omega \times(k T,(k+1) T)
$$

Moreover,

$$
\int_{k T}^{(k+1) T} \int_{\Omega}\left|\alpha\left(\bar{\vartheta}_{m}+\theta_{*}\right)\right|^{2} d x d t \leq c\left(1+\left\|\bar{\vartheta}_{m}\right\|_{L_{2}\left(k T,(k+1) T ; L_{2}(\Omega)\right)}^{2 \sigma}\right) \leq c .
$$

Hence (see [14], Lemma 1.3)

$$
\alpha\left(\bar{\vartheta}_{m}+\theta_{*}\right) \rightarrow \alpha\left(\bar{\vartheta}+\theta_{*}\right) \quad \text { weakly in } L_{2}(\Omega \times(k T,(k+1) T)) .
$$

The last convergence suffices to pass to the limit in the r.h.s. of (27). This way we obtain that $(w, q, \bar{\vartheta})$ is the strong-weak solution to problem (13). This ends the proof of Theorem 1 .

\section{Stability of Two-Dimensional Solution}

To prove the stability of the two-dimensional solution which exists in virtue of Theorem 1 we need some a priori estimates of solutions to problem (3). Assume that

$$
\theta(0) \geq \theta_{*},
$$

where $\theta_{*}>0$ is the constant from assumption (14) and

$$
\left|\alpha^{\prime}(\theta)\right| \leq a_{3} \quad \text { for } \theta \geq \theta_{*},
$$

where $a_{3}>0$ is a constant. First, we formulate the lemma

Lemma 8 Let (32)-(33) and the assumptions of Theorem 1 be satisfied. Assume that $f \in$ $L_{2, \text { loc }}\left(\mathbb{R}_{+} ; L_{\infty}(D)\right)$. Moreover, assume that $(w, \vartheta, q)$ is the two-dimensional solutions of problem (1) which exists in view of Theorem 1 . Let $(u, \chi, \eta)$ be a sufficiently regular solution to problem (3). Then

$$
\begin{aligned}
& \frac{d}{d t}\left(\|\operatorname{rot} u\|_{L_{2}(D)}^{2}+\|\chi\|_{L_{2}(D)}^{2}\right)+c\left(\left\|\operatorname{rot}^{2} u\right\|_{L_{2}(D)}^{2}+\|\chi\|_{H^{1}(D)}^{2}\right) \\
& \leq c\left[\|\operatorname{rot} u\|_{L_{2}(D)}^{6}+\|w\|_{W_{3}^{1}(D)}^{2}\left(\|\operatorname{rot} u\|_{L_{2}(D)}^{2}+\|\chi\|_{L_{2}(D)}^{2}\right)\right. \\
& \quad+\left\|\bar{\vartheta}_{x}\right\|_{L_{2}(D)}^{2}\|u\|_{L_{3}(D)}^{2}+\|\operatorname{rot} u\|_{L_{2}(D)}^{4}\|\chi\|_{L_{2}(D)}^{2}+\|\operatorname{rot} u\|_{L_{2}(D)}^{2}\left\|w_{x}\right\|_{L_{3}(D)}^{2} \\
& \left.\quad+\|\chi\|_{L_{2}(D)}^{2}\|f\|_{L_{\infty}(D)}^{2}+\|g\|_{L_{\infty}(D)}^{2}+\|g\|_{L_{\infty}(D)}^{2}\|\bar{\vartheta}\|_{L_{2}(D)}^{2 \sigma}\right]
\end{aligned}
$$

for $t>0$. 
Proof Multiplying equation (3) $)_{1}$ by $\operatorname{rot}^{2} u$ and integrating over $D$ gives

$$
\begin{aligned}
& \frac{1}{2} \frac{d}{d t}\|\operatorname{rot} u\|_{L_{2}(D)}^{2}+v\left\|\operatorname{rot}^{2} u\right\|_{L_{2}(D)}^{2} \\
& \leq-\int_{D} u \cdot \nabla u \cdot \operatorname{rot}^{2} u d x-\int_{D} w \cdot \nabla u \cdot \operatorname{rot}^{2} u d x-\int_{D} u \cdot \nabla w \cdot \operatorname{rot}^{2} u d x \\
& \quad+\int_{D} \alpha^{\prime}(\zeta) \chi f \operatorname{rot}^{2} u d x+\int_{D} \alpha(\vartheta) g \operatorname{rot}^{2} u d x,
\end{aligned}
$$

where $\zeta=\beta \theta+(1-\beta) \vartheta, \beta \in(0,1)$. Consider

$$
\begin{aligned}
-\int_{D} u \cdot \nabla u \cdot \operatorname{rot}^{2} u d x & =\int_{D} \operatorname{rot}(u \cdot \nabla u) \operatorname{rot} u d x \\
& =\int_{D} u \cdot \nabla \operatorname{rot} u \cdot \operatorname{rot} u d x+\int_{D} \operatorname{rot} u \cdot \nabla u \cdot \operatorname{rot} u \equiv I,
\end{aligned}
$$

where the summation convention over repeated indices is applied. By the boundary conditions

$$
\int_{D} u \cdot \nabla \operatorname{rot} u \cdot \operatorname{rot} u d x=0
$$

so

$$
|I| \leq c\left\|u_{x}\right\|_{L_{3}(D)}^{3} .
$$

Therefore, the Hölder and Young inequalities applied in (35) yield

$$
\begin{aligned}
& \frac{d}{d t}\|\operatorname{rot} u\|_{L_{2}(D)}^{2}+v\left\|\operatorname{rot}^{2} u\right\|_{L_{2}(D)}^{2} \\
& \quad \leq c\left(\left\|u_{x}\right\|_{L_{3}(D)}^{3}+\|w \cdot \nabla u\|_{L_{2}(D)}^{2}+\|u \cdot \nabla w\|_{L_{2}(D)}^{2}\right. \\
& \left.\quad+\|\chi\|_{L_{2}(D)}^{2}\|f\|_{L_{\infty}(D)}^{2}+\|g\|_{L_{\infty}(D)}^{2}+\|g\|_{L_{\infty}(D)}^{2}\|\bar{\vartheta}\|_{L_{2}(D)}^{2 \sigma}\right) .
\end{aligned}
$$

Consider the terms on the right-hand side of (36). First, using the interpolation inequality (see [1])

$$
\left\|u_{x}\right\|_{L_{3}(D)} \leq c\left\|u_{x}\right\|_{H^{1}(D)}^{1 / 2}\left\|u_{x}\right\|_{L_{2}(D)}^{1 / 2}
$$

together with the estimates

$$
\left\|u_{x}\right\|_{L_{2}(D)} \leq c\|u\|_{H^{1}(D)} \leq c\|\operatorname{rot} u\|_{L_{2}(D)}
$$

and

$$
\left\|u_{x}\right\|_{H^{1}(D)} \leq c\|\operatorname{rot} u\|_{H^{1}(D)} \leq c\left\|\operatorname{rot}^{2} u\right\|_{L_{2}(D)}
$$

(see Lemma 3) we have

$$
\left\|u_{x}\right\|_{L_{3}(D)}^{3} \leq \varepsilon\left\|\operatorname{rot}^{2} u\right\|_{L_{2}(D)}^{2}+c(\varepsilon)\|\operatorname{rot} u\|_{L_{2}(D)}^{6},
$$


where the Young inequality has been also used. Next,

$$
\|w \cdot \nabla u\|_{L_{2}(D)}^{2} \leq c\|w\|_{L_{\infty}(D)}^{2}\|\nabla u\|_{L_{2}(D)}^{2} \leq c\|w\|_{W_{3}^{1}(D)}^{2}\|\nabla u\|_{L_{2}(D)}^{2},
$$

where we used that $w$ only depends on $x_{1}, x_{2}$. Finally, the third term on the right-hand side of (36) is bounded by

$$
c\|u\|_{L_{6}(D)}^{2}\|\nabla w\|_{L_{3}(D)}^{2} \leq c\|u\|_{H^{1}(D)}^{2}\|\nabla w\|_{L_{3}(D)}^{2} .
$$

Hence inequality (36) yields

$$
\begin{aligned}
& \frac{d}{d t}\|\operatorname{rot} u\|_{L_{2}(D)}^{2}+c\left\|\operatorname{rot}^{2} u\right\|_{L_{2}(D)}^{2} \\
& \quad \leq c\left(\|\operatorname{rot} u\|_{L_{2}(D)}^{6}+\|w\|_{W_{3}^{1}(D)}^{2}\|u\|_{H^{1}(D)}^{2}\right. \\
& \left.\quad+\|\chi\|_{L_{2}(D)}^{2}\|f\|_{L_{\infty}(D)}^{2}+\|g\|_{L_{\infty}(D)}^{2}+\|g\|_{L_{\infty}(D)}^{2}\|\bar{\vartheta}\|_{L_{2}(D)}^{2 \sigma}\right) .
\end{aligned}
$$

Now, multiply (3) $)_{3}$ by $\chi$ and integrate the result over $D$. We get

$$
\begin{aligned}
& \frac{1}{2} \frac{d}{d t}\|\chi\|_{L_{2}(D)}^{2}+\varkappa\|\nabla \chi\|_{L_{2}(D)}^{2} \\
& \quad=-\int_{D} w \cdot \nabla \chi \chi d x-\int_{D} u \cdot \nabla \bar{\vartheta} \chi d x+v \int_{D}|\mathbb{D}(u)|^{2} \chi d x+2 v \int_{D} \mathbb{D}(u): \mathbb{D}(w) \chi d x .
\end{aligned}
$$

The first term on the right-hand side of (40) is bounded by

$$
\varepsilon\|\nabla \chi\|_{L_{2}(D)}^{2}+c(\varepsilon)\|w\|_{L_{\infty}(D)}^{2}\|\chi\|_{L_{2}(D)}^{2} \leq \varepsilon\|\nabla \chi\|_{L_{2}(D)}^{2}+c(\varepsilon)\|w\|_{W_{3}^{1}(D)}^{2}\|\chi\|_{L_{2}(D)}^{2} .
$$

The second term is estimated by

$$
\varepsilon\|\chi\|_{L_{6}(D)}^{2}+c(\varepsilon)\|\nabla \bar{\vartheta}\|_{L_{2}(D)}^{2}\|u\|_{L_{3}(D)}^{2} .
$$

We estimate the third term as follows

$$
\begin{aligned}
\int_{D}|\mathbb{D}(u)|^{2} \chi d x & \leq\left\|u_{x}\right\|_{H^{1}(D)}\left\|u_{x}\right\|_{L_{2}(D)}\|\chi\|_{L_{3}(D)} \\
& \leq \varepsilon\left\|u_{x}\right\|_{H^{1}(D)}^{2}+c(\varepsilon)\left\|u_{x}\right\|_{L_{2}(D)}^{2}\|\chi\|_{L_{3}(D)}^{2} \\
& \leq \varepsilon\left\|u_{x}\right\|_{H^{1}(D)}^{2}+c(\varepsilon)\left\|u_{x}\right\|_{L_{2}(D)}^{2}\|\chi\|_{H^{1}(D)}\|\chi\|_{L_{2}(D)} \\
& \leq \varepsilon\left(\left\|u_{x}\right\|_{H^{1}(D)}^{2}+\left\|\chi_{x}\right\|_{L_{2}(D)}^{2}\right)+c(\varepsilon)\left\|u_{x}\right\|_{L_{2}(D)}^{4}\|\chi\|_{L_{2}(D)}^{2},
\end{aligned}
$$

where the interpolation inequality

$$
\|\chi\|_{L_{3}(D)} \leq c\|\chi\|_{H^{1}(D)}^{1 / 2}\|\chi\|_{L_{2}(D)}^{1 / 2}
$$

has been used (see [1]). Finally,

$$
\int_{D} \mathbb{D}(u): \mathbb{D}(w) \chi d x \leq \varepsilon\|\chi\|_{L_{6}(D)}^{2}+c(\varepsilon)\left\|u_{x}\right\|_{L_{2}(D)}^{2}\left\|w_{x}\right\|_{L_{3}(D)}^{2} .
$$


Using the above estimates in (36) we obtain

$$
\begin{aligned}
\frac{d}{d t}\|\chi\|_{L_{2}(D)}^{2}+c\|\nabla \chi\|_{L_{2}(D)}^{2} \leq & \varepsilon\left(\left\|u_{x}\right\|_{H^{1}(D)}^{2}+\left\|\chi_{x}\right\|_{L_{2}(D)}^{2}+\|\chi\|_{L_{6}(D)}^{2}\right) \\
& +c(\varepsilon)\left(\|w\|_{W_{3}^{1}(D)}^{2}\|\chi\|_{L_{2}(D)}^{2}+\|\nabla \bar{\vartheta}\|_{L_{2}(D)}^{2}\|u\|_{L_{3}(D)}^{2}\right. \\
& \left.+\left\|u_{x}\right\|_{L_{2}(D)}^{4}\|\chi\|_{L_{2}(D)}^{2}+\left\|u_{x}\right\|_{L_{2}(D)}^{2}\left\|w_{x}\right\|_{L_{3}(D)}^{2}\right) .
\end{aligned}
$$

Inequalities (37)-(39) and (41) imply (34).

Introduce the notation

$$
\begin{aligned}
& X(t)=\|\operatorname{rot} u(t)\|_{L_{2}(D)}^{2}+\|\chi(t)\|_{L_{2}(D)}^{2}, \\
& A(t)=\|w(t)\|_{W_{3}^{1}(D)}^{2}+\left\|\vartheta_{x}(t)\right\|_{L_{2}(D)}^{2}+\|f(t)\|_{L_{\infty}(D)}^{2}, \\
& G(t)=\|g(t)\|_{L_{\infty}(D)}^{2} .
\end{aligned}
$$

By Lemma 6

$$
\begin{aligned}
& \int_{k T}^{t} A\left(t^{\prime}\right) d t^{\prime} \leq \tilde{c}_{0}\left(A_{1}+A_{2}+\sup _{k \in \mathbb{N}_{0}} \int_{k T}^{(k+1) T}\|f\|_{L_{\infty}(D)}^{2} d t\right) \equiv A_{3}(T) \\
& \quad \text { for all } t \in(k T,(k+1) T], k \in \mathbb{N}_{0},
\end{aligned}
$$

where $\tilde{c}_{0}>0$ does not depend on $k$.

Lemma 9 Let the assumptions of Lemma 8 hold. Assume that

$$
\frac{\tilde{c}_{1} T}{4}>\tilde{c}_{2} A_{3}(T),
$$

where $\tilde{c}_{1}, \tilde{c}_{2}>0$ are the constants from (48). Let $\gamma$ and $\delta$ be so small that

$$
\tilde{c}_{2} M \gamma^{2}+\delta \leq \frac{\tilde{c}_{1}}{2}
$$

where $M>0$ is the constant from (49). Moreover, let $T$ be so large and $\delta$ be so small that

$$
e^{-\frac{\tilde{c}_{1}}{4} T}+M T \delta \leq 1 .
$$

If $X(0) \leq \gamma$ and $G(t) \leq \delta \gamma$ for all $t \in \mathbb{R}_{+}$, then $X(t) \leq \tilde{c} \gamma$ for all $t \in \mathbb{R}_{+}$, where $\tilde{c}=\tilde{c}(T)$ does not depend on $k$.

Proof By Lemma 5

$$
\sup _{t \in[k T,(k+1) T]}\|\bar{\vartheta}\|_{L_{2}(D)}^{2 \sigma} \leq c\left(A_{1}+A_{2}\right) \quad \text { for all } k \in \mathbb{N}_{0},
$$

where $c>0$ does not depend on $k$. Now, by (42) and estimate (47), inequality (34) can be written in the form

$$
\frac{d X}{d t}+\tilde{c}_{1} X \leq \tilde{c}_{2}\left(X^{3}+A X+G\right) \quad \text { for } t \in(k T,(k+1) T], k \in \mathbb{N}_{0} .
$$


Hence

$$
\begin{aligned}
& \frac{d Z}{d t}+\tilde{c}_{1} Z \leq \tilde{c}_{2}\left(e^{2 \tilde{c}_{2} \int_{k T}^{t} A\left(t^{\prime}\right) d t^{\prime}} Z^{3}+e^{-\tilde{c}_{2} \int_{k T}^{t} A\left(t^{\prime}\right) d t^{\prime}} G\right) \\
& \quad \text { for } t \in(k T,(k+1) T], \quad k \in \mathbb{N}_{0},
\end{aligned}
$$

where

$$
Z(t)=e^{-\tilde{c}_{2} \int_{k T}^{t} A\left(t^{\prime}\right) d t^{\prime}} X(t)
$$

Inequality (43) implies

$$
e^{2 \tilde{c}_{2} \int_{k T}^{t} A\left(t^{\prime}\right) d t^{\prime}} \leq M^{2} \quad \text { for all } t \in(k T,(k+1) T], \quad k \in \mathbb{N}_{0},
$$

where $M>0$ is some constant which does not depend on $k$. Therefore

$$
\frac{d Z}{d t}+\tilde{c}_{1} Z \leq \tilde{c}_{2}\left(M^{2} Z^{3}+G\right) \quad \text { for all } t \in(k T,(k+1) T], k \in \mathbb{N}_{0} .
$$

Assume that

$$
X(k T) \leq \gamma \quad \text { for some } k \in \mathbb{N}_{0}
$$

We will show that

$$
X((k+1) T) \leq \gamma
$$

Inequality (50) implies that

$$
Z(k T) \leq \gamma .
$$

We will prove that $Z(t) \leq \gamma$ for all $t \in[k T,(k+1) T]$. Let $t^{*}=\inf \{t: Z(t)>\gamma\}$. Then

$$
\frac{d Z}{d t}\left(t^{*}\right)+\tilde{c}_{1} \gamma \leq \tilde{c}_{2}\left(M^{2} \gamma^{3}+\delta \gamma\right)
$$

By assumption (45) we have

$$
\frac{d Z}{d t}\left(t^{*}\right)<0,
$$

so $Z(t) \leq \gamma$ for all $t \in[k T,(k+1) T]$. This implies that

$$
X(t) \leq \gamma M \quad \text { for all } t \in(k T,(k+1) T] .
$$

Using (52) in (48) we get

$$
\frac{d X}{d t}+\tilde{c}_{1} X \leq \tilde{c}_{2}\left(\gamma^{2} M^{2} X+A X+G\right) \quad \text { for all } t \in(k T,(k+1) T]
$$

and (45) yields

$$
\frac{d X}{d t}+\frac{\tilde{c}_{1}}{2} X \leq \tilde{c}_{2}(A X+G) \quad \text { for all } t \in(k T,(k+1) T] .
$$

Hence

$$
\frac{d}{d t}\left(e^{\frac{\tilde{c}_{1}}{2} t-\tilde{c}_{2} \int_{k T}^{t} A\left(t^{\prime}\right) d t^{\prime}} X\right) \leq e^{\frac{\tilde{c}_{1}}{2} t-\tilde{c}_{2} \int_{k T}^{t} A\left(t^{\prime}\right) d t^{\prime}} G .
$$


After integrating the above inequality with respect to $t$ from $t=k T$ to $t \in(k T,(k+1) T]$ we obtain

$$
\begin{aligned}
& X(t) \leq e^{-\frac{\tilde{c}_{1}}{2}(t-k T)+\tilde{c}_{2} \int_{k T}^{t} A\left(t^{\prime}\right) d t^{\prime}} X(k T)+e^{-\frac{\tilde{c}_{1}}{2} t+\tilde{c}_{2} \int_{k T}^{t} A\left(t^{\prime}\right) d t^{\prime}} \int_{k T}^{t} e^{\frac{\tilde{c}_{1}}{2} t^{\prime}-\tilde{c}_{2} \int_{k T}^{t^{\prime}} A\left(t^{\prime \prime}\right) d t^{\prime \prime}} G\left(t^{\prime}\right) d t^{\prime} \\
& \text { for } t \in(k T,(k+1) T] .
\end{aligned}
$$

For $t=(k+1) T$ we have

$$
X((k+1) T) \leq e^{-\frac{\tilde{c}_{1}}{2} T+\tilde{c}_{2} \int_{k T}^{(k+1) T} A(t) d t} X(k T)+e^{\tilde{c}_{2} \int_{k T}^{(k+1) T} A\left(t^{\prime}\right) d t^{\prime}} \int_{k T}^{(k+1) T} G\left(t^{\prime}\right) d t^{\prime} .
$$

Now, assumption (44) and estimate (43) imply

$$
\frac{\tilde{c}_{1} T}{4}>\tilde{c}_{2} \int_{k T}^{t} A\left(t^{\prime}\right) d t^{\prime} \quad \text { for } t \in(k T,(k+1) T] .
$$

Hence

$$
X((k+1) T) \leq e^{-\frac{\tilde{c}_{1} T}{4}} X(k T)+M \int_{k T}^{(k+1) T} G(t) d t .
$$

Now, (50), (46) and the assumption $G(t) \leq \delta \gamma$ for all $t \in \mathbb{R}_{+}$yield (51). Therefore, we showed that $X(k T) \leq \gamma$ for all $k \in \mathbb{N}_{0}$. Next, by (53) we get

$$
\begin{aligned}
& X(t) \leq M \gamma+M \int_{k T}^{(k+1) T} G\left(t^{\prime}\right) d t^{\prime} \leq M \gamma(1+\delta T) \equiv \tilde{c} \gamma \\
& \text { for all } t \in(k T,(k+1) T], k \in \mathbb{N}_{0} .
\end{aligned}
$$

This ends the proof.

Lemma 10 Let the assumptions of Lemma 9 be satisfied. Moreover, assume that

$$
\sup _{k \in \mathbb{N}_{0}} \int_{k T}^{(k+1) T}\|f(t)\|_{L_{\infty}(D)}^{2} d t<\infty .
$$

Then the following estimate holds

$$
\begin{aligned}
& \|u\|_{H^{2,1}(D \times(k T,(k+1) T))}^{2}+\|\chi\|_{L_{2}\left(k T,(k+1) T ; H^{1}(D)\right)}^{2}+\left\|\chi_{t}\right\|_{L_{2}\left(k T,(k+1) T ; H^{-1}(D)\right)}^{2} \\
& \quad+\|\nabla q\|_{L_{2}\left(k T,(k+1) T ; L_{2}(D)\right)}^{2} \leq c(T) \gamma
\end{aligned}
$$

Proof Integrating (34) with respect to time from $k T$ to $t \in[k T ;(k+1) T]$ and using estimates (37)-(38) yields

$$
\|u\|_{L_{2}\left(k T, t ; H^{2}(D)\right)}^{2}+\|\chi\|_{L_{2}\left(k T, t ; H^{1}(D)\right)}^{2} \leq c(T) \gamma
$$


for $t \in[k T,(k+1) T], \quad k \in \mathbb{N}_{0}$. Now, estimate (8) implies the estimates

$$
\begin{aligned}
\left\|u_{t}\right\|_{L_{2}\left(k T,(k+1) T ; L_{2}(D)\right)}^{2} & \leq c(T) \gamma, \\
\|\nabla \eta\|_{L_{2}\left(k T,(k+1) T ; L_{2}(D)\right)}^{2} & \leq c(T) \gamma
\end{aligned}
$$

and

$$
\left\|\chi_{t}\right\|_{L_{2}\left(k T,(k+1) T ; H^{-1}(D)\right)}^{2} \leq c(T) \gamma \quad \text { for all } k \in \mathbb{N}_{0} .
$$

This completes the proof.

The proofs of Theorems 2 and 3 The assertion of Theorem 2 is the immediate consequence of Lemmas 8-10. The assertion of Theorem 3 follows by using estimates of Lemmas 9-10 and the Faedo-Galerkin method.

Open Access This article is distributed under the terms of the Creative Commons Attribution 4.0 International License (http://creativecommons.org/licenses/by/4.0/), which permits unrestricted use, distribution, and reproduction in any medium, provided you give appropriate credit to the original author(s) and the source, provide a link to the Creative Commons license, and indicate if changes were made.

\section{References}

1. Besov, O.V., Il'in, V.P., Nikol'skii, S.M.: Integral Representations of Functions and Imbedding Theorems. Nauka, Moscow (1975) (in Russian)

2. Brandolese, L., Schonbek, M.E.: Large time decay and growth for solutions of a viscous Boussinesq system. Trans. Am. Math. Soc. 364, 5057-5090 (2012)

3. Buliček, M., Feireisl, E., Málek, J.: A Navier-Stokes-Fourier system for incompressible fluids with temperature dependent material coefficients. Nonlinear Anal., Real World Appl. 10, 992-1015 (2009)

4. Buliček, M., Kaplicky, P., Málek, J.: An $L^{2}$-maximal regularity result for the evolutionary Stokes-Fourier system. Appl. Anal. 90, 31-45 (2011)

5. Consighlieri, L., Rodrigues, J.F., Shilkin, T.: On the Navier-Stokes equations with energy-dependent nonlocal viscosities. J. Math. Sci. 130, 4814-4826 (2005)

6. Diaz, J.I., Galiano, G.: Existence and uniqueness of solutions of the Boussinesq system with nonlinear thermal diffusion. Topol. Methods Nonlinear Anal. 11, 59-82 (1998)

7. Feireisl, E., Málek, J.: On the Navier-Stokes equations with temperature-dependent transport coefficients. Differ. Equ. Nonlinear Mech. 2006, 90616 (2006) (electronic), 14 pp.

8. Goncharova, O.N.: Solvability of the nonstationary problem for the equations of free convection with temperature dependent viscosity. Din. Sploš. Sredy 96, 35-58 (1990) (in Russian)

9. Goncharova, O.N.: Unique solvability of a two-dimensional nonstationary problem for the convection equations with temperature-dependent viscosity. Differ. Uravn. 38, 234-242 (2002) (in Russian). English translation: Differ. Equ. 38, 249-258 (2002)

10. Kagei, Y.: On weak solutions of nonstationary Boussinesq equations. Differ. Integral Equ. 6, 587-611 (1993)

11. Kagei, Y.: Atractors of two-dimensional equations of thermal convection in the presence of the dissipation function. Hiroshima Math. J. 25, 251-311 (1995)

12. Kagei, Y.: On two-dimensional equations of thermal convection in the presence of the dissipation function. In: Theory of the Navier-Stokes Equations. Series on Advances in Mathematics for Applied Sciences, vol. 47, pp. 72-85. World Scientific, Singapore (1998)

13. Kagei, Y., Ružička, M., Thäter, G.: Natural convection with dissipative heating. Commun. Math. Phys. 214, 287-313 (2000)

14. Lions, J.L.: Quelques Methodes de Résolution des Problémes aux Limites non Linéaires. Dunod, Paris (1969)

15. Liu, X., Li, Y.: On the stability of global solutions to the 3d Boussinesq system. Nonlinear Anal. 95, 580-591 (2014)

16. Morimoto, H.: Non-stationary Boussinesq equations. J. Fac. Sci., Univ. Tokyo, Sect. 1A, Math. 39, 61-75 (1992) 
17. Naumann, J.: On the existence of weak solutions to the equations of non-stationary motion of heatconducting incompressible viscous fluids. Math. Methods Appl. Sci. 29, 1883-1903 (2006)

18. Shilkin, T.: Classical solvability of the coupled system modelling a heat-convergent Poiseuille-type flow. J. Math. Fluid Mech. 7, 72-84 (2005)

19. Socała, J., Zajączkowski, W.M.: Long time existence of regular solutions to $3 \mathrm{~d}$ Navier-Stokes equations coupled with heat convection. Appl. Math. 30, 231-242 (2012)

20. Solonnikov, V.A.: Overdetermined elliptic boundary value problems. Zap. Nauch. Semin. LOMI 21, 112-158 (1971) (in Russian)

21. Zadrzyńska, E., Zajączkowski, W.M.: Stability of two-dimensional Navier-Stokes motions in the periodic case. J. Math. Anal. Appl. 423, 956-974 (2015)

22. Zadrzyńska, E., Zajączkowski, W.M.: Stability of two-dimensional heat-conducting incompressible motions in a cylinder. Nonlinear Anal. 125, 113-127 (2015)

23. Zajączkowski, W.M.: Stability of two-dimensional solutions to the Navier-Stokes equations in cylindrical domains under Navier boundary condition. J. Math. Anal. Appl. 444(1), 275-297 (2016) 